\title{
ALUMINUM NITRIDE VICINITY-BASED RESONANT CHARGING LINK
}

\author{
E. Mehdizadeh and G. Piazza
}

\author{
Carnegie Mellon University, Pittsburgh, Pennsylvania, USA
}

\begin{abstract}
This work reports on a low-cost and CMOS compatible approach for the synthesis of a through package charging and authenticating communication link enabled by GHz-range aluminum nitride (AlN) piezoelectric transducers. The acoustic link is formed by two acoustic transducers that leverage the enhancement of energy transfer at resonance. Power transfer efficiency (PTE) of $-12 \mathrm{~dB}$ in agreement with the simulation results is measured for a transducer size of $1 \times 100 \times 100 \mu \mathrm{m}^{2}$ through a $700 \mu \mathrm{m}$ thick silicon substrate. This work is the first step towards the development of next-generation high-efficiency and micro-scale power transfer units that could energize chips through packages or biomedical implants.
\end{abstract}

\section{INTRODUCTION}

The security and integrity of electronic systems is challenged by the presence of counterfeit integrated circuits (ICs) in the supply chain. Counterfeiters use a variety of easy and inexpensive techniques to recycle discarded ICs, alter them, and reintroduce them to the supply chain for profit. Introducing vicinity-based authenticating communication and charging capability into microscopic-scale chips that can be inserted into temper-resist packaging of ICs can be a viable solution for eliminating the prospect of counterfeiting from the electronic supply chain. Furthermore, near-field wireless powering of microsystems operating in liquid [1] or embedded in hazardous and inaccessible areas such as the human body [2] or sealed vessels [3] could be of great interest as frequent replacement of battery sources in such systems is cumbersome and expensive.

When dealing with metallic packages, the use of acoustic waves to power any IC is the only option as electromagnetic waves would decay exponentially into conductive materials [4]. Moreover, the use of acoustic transducers ensures higher PTE than any other wireless links over a distance that is $10 \sim 100$ times larger than the acoustic transducer dimensions. Acoustic or ultrasound power transfer has been previously studied mostly for high power industrial applications operating at low frequencies [5]. Chip-scale acoustic resonators, with the same working principle but operating at higher/RF frequencies, have also been extensively researched in the MEMS field for frequency synthesis and selection [6]. However, chip-scale acoustic power transfer is yet to be fully explored. Due to record-breaking $f-Q$ products up to $10^{14}$, high overtone bulk acoustic resonators (HBARs) [7] represent ideal candidates for the realization of power transfer units whose efficiency is dramatically enhanced by operation at resonance. Since fabrication of such structures requires no releasing step, they represent extremely robust solutions for various applications. Through substrate acoustic links have been previously demonstrated with HBAR devices based on different piezoelectric materials [8], [9], validating the high efficiency and low loss feature of such acoustic links. Hoople et al. [10] also reported the use of aluminum nitride transducers for on chip communication by sending an acoustic pulse from one transducer in an array, reflecting the pulse off the bottom surface of the silicon substrate, and receiving the pulse from a neighboring transducer in the array. Kuo et al [11] then took the concept one step further and used aluminum nitride transducers in a Through-SiliconVia (TSV)-like communication link. However, none of these device have been applied to through packaging power transfer and charging. In this work, we introduce chip-scale acoustic transducers

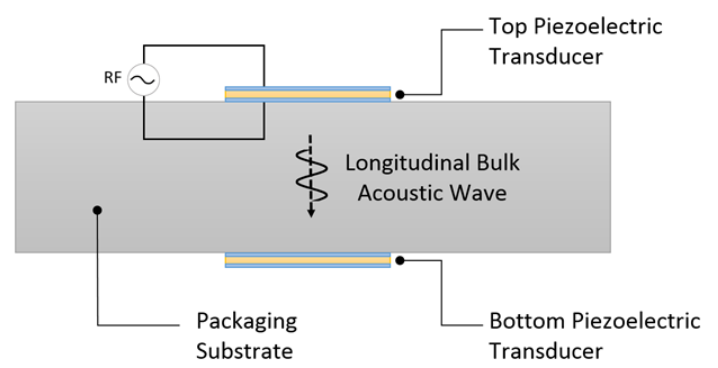

Figure 1: Schematic presentation of the HBAR-like acoustic link used for through package power transfer.

in a two-port configuration as promising solutions for throughpackage energy transfer and charging. As a proof of concept, herein, we experimentally validate the acoustic link on double side polished single crystalline silicon substrates and prove, through simulations, how the approach can be extended to other materials.

\section{DEVICE DESCRIPTION AND FABRICATION}

The presented platform consists of two identical thin piezoelectric films each sandwiched by two metallic electrodes mounted on the two sides of a substrate (Figure 1). The longitudinal bulk acoustic waves (BAW) that are originated by vibrations of the top piezoelectric acoustic transducer propagate through the acoustic channel (the silicon substrate in this implementation) and are absorbed by an identical transducer on the other side of the package. Similar to FBARs, the individual transducers utilized here can be excited to vibrate across the thickness of the film (see Figure 2). The main piezoelectric coefficient used to excite the thickness mode vibrations is $d_{33}$. As a result, thickness mode vibrations can result in high electromechanical coupling making the technology suitable for broadband power transfer. The film thickness, $t$, sets the resonance frequency of the device for a given overall acoustic velocity of the material stack, $c_{\mathrm{ac}}$ (piezoelectric and metal films).

$$
f_{0}=\frac{1}{2 t} c_{a c}
$$

Aluminum nitride is elected here as a CMOS-compatible and high electromechanical piezoelectric material. The fabrication process also only involves standard deposition and etching steps to ensure low cost. As a proof of concept, the devices are fabricated on a $700 \mu \mathrm{m}$ thick high resistivity double side polished silicon substrate. The process starts with sputtering and patterning of 100 $\mathrm{nm}$ thick Pt layers (bottom electrode) on both sides of the wafer using lift-off (Fig. 3a). $1 \mu \mathrm{m}$ thick aluminum nitride layers are then deposited using sputtering and patterned via $\mathrm{Cl}_{2} / \mathrm{BCl}_{3} / \mathrm{Ar}$ based dry etch to access the bottom electrodes (Fig. 3b). In the next step, another $100 \mathrm{~nm}$ thick Pt layer is deposited and patterned on both sides to define the transducers' geometries (Fig. 3c). Finally, the electrical pads are coated with $300 \mathrm{~nm}$ thick gold layer (Fig. 3d) to enable flip chip bonding as well as to ensure good contact with the probes. For maximum performance, it is imperative to carefully control the thickness of AlN and Pt films on both sides to match so that the two transducers have close resonance frequencies. For instance, about $50 \mathrm{~nm}$ difference in the thicknesses of $3 \mathrm{GHz}$ AlN transducer films results in $\sim 40 \mathrm{MHz}$ difference in their frequencies and $\sim 1 \mathrm{~dB}$ degradation in overall PTE. Figure 4 depicts the optical microscopic images of the fabricated top and bottom transducers. 


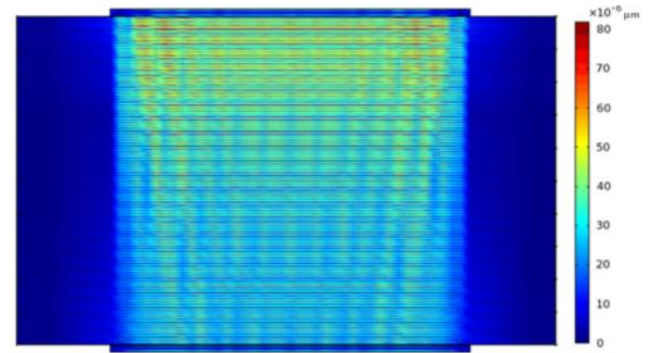

Figure 2: COMSOL displacement mode shape.

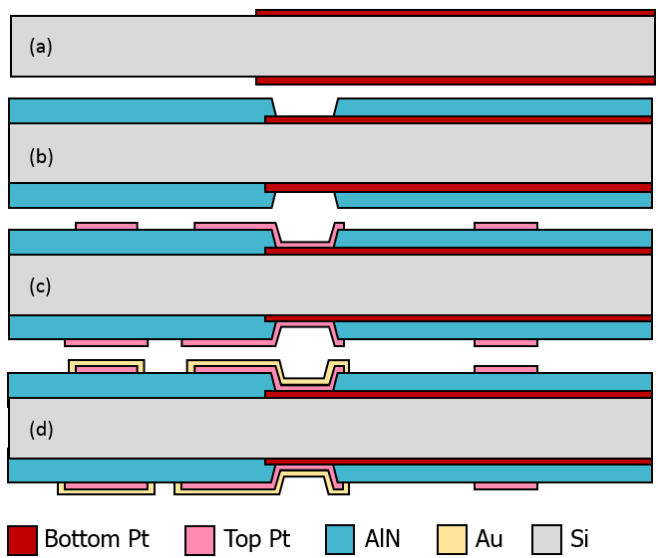

Figure 3: Eight-mask CMOS compatible fabrication process.

The interesting aspect of such platform is that it does not involve any released parts, hence dramatically enhancing yields and making the structure more robust to any environment. Even though the top and bottom transducer geometries are identical, the two structures have different interconnects and traces to enable GSG probing on the top transducer and flip chip bonding on the bottom transducer.

\section{DEVICE MODELING}

The structure is formed by two equivalent transducers and is tested in a two-port configuration. The equivalent electromechanical model of the acoustic link can be obtained by means of connecting the output and input ports of the equivalent models of adjacent layers (Fig. 5). Taking advantage of Mason's model, electrode and packaging layers can be represented by two-port circuits, while three-port circuits are used for piezoelectric slabs [12]. As shown in the figure, the structure is modelled as a three electrical terminal device (effectively four, two of which are shorted together to ground), representing input, output, and ground. In Fig. 5, force, $F$, and acoustic velocity, $v$, components on the two sides of each layer play a role similar to voltage and current in an electrical circuit. The governing equations between force and velocity components can then be expressed as:

$\left\{F_{n_{i 1}}=a_{n} v_{n_{i 1}}+b_{n}\left(v_{n_{i 1}}+v_{n_{i 2}}\right)\right.$

$\left\{\begin{array}{l}F_{n_{i 2}}=a_{n} v_{n_{i 2}}+b_{n}\left(v_{n_{i 1}}+v_{n_{i 2}}\right)\end{array} \quad n=p, m, a\right.$

where $p, m$, and $a$ subscripts denote piezoelectric, metal, and packaging media, respectively. $a_{n}$ and $b_{n}$ are frequency dependent and can be written as:

$\left\{\begin{array}{c}a_{n}=i Z_{n} \tan \left(\frac{k_{n} d_{n}}{2}\right) \\ b_{n}=-i Z_{n} \sin \left(k_{n} d_{n}\right)\end{array}\right.$

where $Z_{n}$ is the characteristic acoustic impedance of the layers, $d_{n}$ is
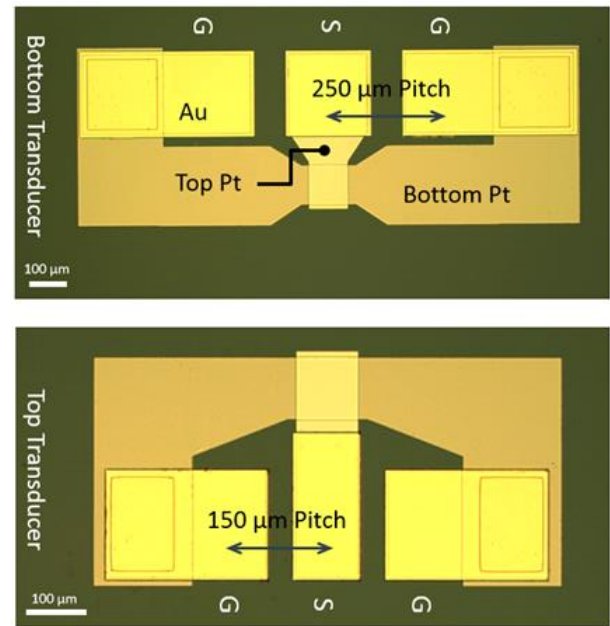

Figure 4: Optical microscopic images of the top and bottom transducers.

the layer thickness, and $k_{n}$ is the wave number which is defined as:

$k_{n}=\omega / c_{n}-j \alpha_{n}$

where $\omega, c$, and $\alpha$ are angular frequency, velocity of sound, and the acoustic attenuation coefficient. Attenuation coefficient lumps all kind of losses in the transducer as well as the packaging material. Since the attenuation coefficient tends to proportionally increase with frequency for most of materials, larger acoustic losses are expected at higher frequencies. However, it should be noted that due to the simplicity of the fab, $\sim 3 \mathrm{GHz}$ resonators employing $1 \mu \mathrm{m}$ thick AlN films are demonstrated in this work. Thicker films, up to $6 \mu \mathrm{m}$ [13], could be used to lower the device center frequency.

For the piezoelectric slabs a further force term is added to the right side of equation (2):

$f_{p_{i}}=h C_{0}\left(V_{i}-\frac{h\left(v_{p_{i 1}}+v_{p_{i 2}}\right)}{i \omega}\right)$

where $h$ and $C_{0}$ are the piezoelectric constant and static capacitance of the rigidly attached transducer. When the material is piezoelectric, a third equation should also count for the electrical port:

$I_{i}=i \omega C_{0} V_{i}-h C_{0}\left(v_{p_{i 1}}+v_{p_{i 2}}\right)$

When both the source and load are matched with the system impedance ( $50 \Omega$ in most cases), the power transfer efficiency (PTE) can be calculated using the following equation:

$G_{T}=\left|S_{21}\right|^{2}$

By analyzing the equivalent electromechanical model via Advanced Design Systems (ADS) S-parameter simulations and assuming perfect alignment between the two transducers, PTE values for $700 \mu \mathrm{m}$ thick channels of different media at the center frequencies of $2.7 \mathrm{GHz}$ and $500 \mathrm{MHz}$ can be predicted as summarized in Table 1 . It can be seen from Table 1 that by simply increasing the film thickness, lower operating frequencies and hence better PTEs can be achieved. Clearly, the acoustic link efficiency benefits by using lower center frequency at the expenses of bandwidth. In this manuscript, our goal is to present the general model and validate the principle of operation of the acoustic link. Trade-offs between PTE, bandwidth, and robustness will have to be considered depending on the specific application. 


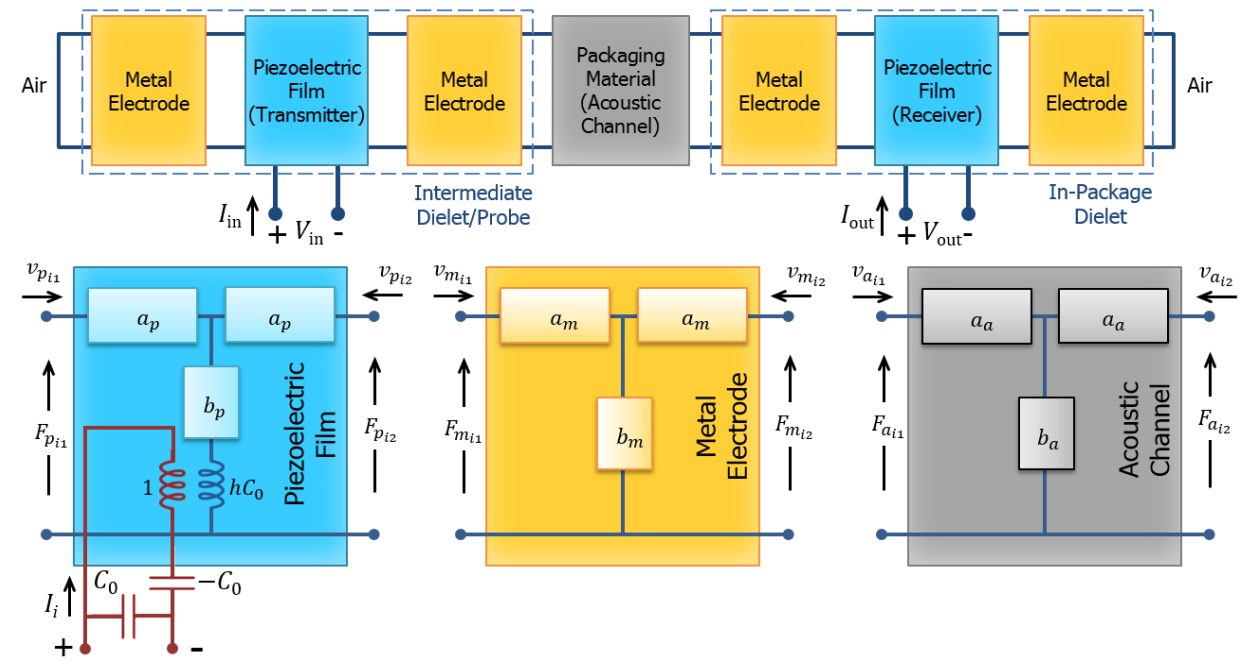

Figure 5: Equivalent electromechanical circuit of the acoustic channel.

Table 1: Simulated PTE prediction for other packaging materials at two different operating frequencies.

\begin{tabular}{ccccc}
\hline $\begin{array}{c}\text { Packaging } \\
\begin{array}{c}\text { Material } \\
(\mathbf{7 0 0} \boldsymbol{\mu m} \\
\text { Thick) }\end{array}\end{array}$ & $\begin{array}{c}\text { Attenuation } \\
\text { Coefficient }(\alpha) \\
\text { @2.7 GHz, } \\
\mathbf{d B} / \mathbf{c m}\end{array}$ & $\begin{array}{c}\text { PTE (dB) } \\
\text { for } \mathbf{1} \boldsymbol{\mu m} \text { AIN, } \\
\mathbf{5 0 ~} \mathbf{~ h m ~ L o a d ~}\end{array}$ & $\begin{array}{c}\text { Attenuation } \\
\text { Coefficient }(\alpha) \\
\text { @500 MHz, } \\
\mathbf{d B} / \mathbf{c m}\end{array}$ & $\begin{array}{c}\text { PTE (dB) } \\
\text { for } \mathbf{~} \boldsymbol{\mu m} \text { AIN, } \\
\mathbf{5 0 ~ O h m ~ L o a d ~}\end{array}$ \\
\hline Rexolite $^{\bullet}$ & 950 & -68.0 & 176 & -13.0 \\
\hline Aluminum & 55 & -18.2 & 10.2 & -3.4 \\
\hline Glass & 18 & -13.5 & 3.4 & -2.5 \\
\hline Silicon & 11 & -11.4 & 2 & -2.1 \\
\hline
\end{tabular}

\section{EXPERIMENTS}

As a proof of concept and in order to demonstrate the predicted PTE values, identical transducers with less than $1 \%$ variations in their thicknesses were realized on double side polished silicon substrate and diced into $3 \times 3 \mathrm{~mm}^{2}$ dies. As illustrated in Figure 4, the transducer design was constrained to a maximum size of $100 \times 100 \mu \mathrm{m}^{2}$ because of the specific IC application it targeted. To test the devices in the two-port configuration, the top and bottom transducers were designed for direct GSG probing and flip chip bonding with another silicon carrier chip, respectively. Gold patterned carrier substrate to be bonded with the backside transducers were fabricated on high resistivity silicon substrates using a specifically designed mask. The dimensions of the carrier silicon chips after dicing is $7 \times 7 \mathrm{~mm}^{2}$. Flip chip bonding was conducted in house using a Laurier M9 bonder. Direct gold to gold bonds were created through application of $1000 \mathrm{gr}$ force between the two substrates heated up to $200{ }^{\circ} \mathrm{C}$. Such bonds are produced without application of any conductive adhesive material. As it can be seen from Figure 6, to enable simultaneous probing of the bottom along with the top transducer, long gold traces had to be extended from the center of the chips to the open area of the bottom die. To probe the bottom transducers in the two-port configuration, the GSG probes land on the testbed silicon substrate (see Figure 7). In operation, the input RF signal of the network analyzer excites the AlN thin film on the top transducer into the thickness mode vibration creating longitudinal bulk acoustic waves that propagate through the silicon substrate and are received by the bottom transducer. The transducers performed as expected at $3 \mathrm{GHz}$ showing a PTE of $-12 \mathrm{~dB}$ when terminated with $50 \Omega$ (Figure 8 and 9). As it can be seen from the figures, the experimental results agree well with the predicted values obtained from the analytical model.

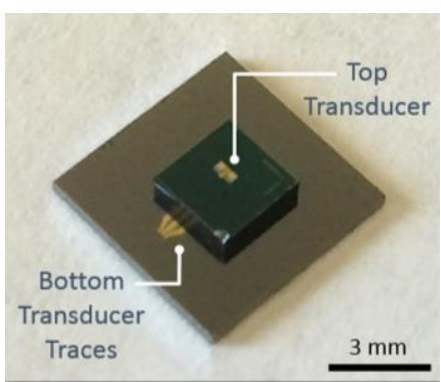

Figure 6: Custom-made measurement setup via flip-chip bonding technique.

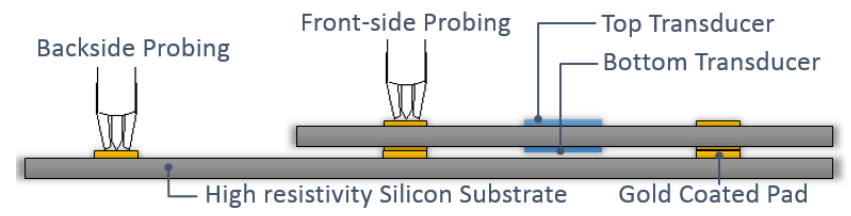

Figure 7: Custom-made measurement setup via flip-chip bonding technique.

It should be also noted that slightly better PTE values $(\sim 1 \mathrm{~dB})$ can be achieved if the load and the source are terminated with optimized impedance values ( $30 \Omega$ for this specific implementation).

The periodic nature of the frequency response is due to the packaging substrate that operates as a resonant cavity. Essentially when the substrate is of finite thickness, the resonant response is modulated by the effects of resonance in this cavity and the response of the piezoelectric layer becomes an envelope of all the harmonics due to the substrate. $6 \mathrm{MHz}$ frequency separation of the harmonics is dictated by the velocity of the elastic waves and thickness of the packaging medium as

$\Delta f=\frac{v_{a}}{2 d_{a}}$

The quality factor, which is extracted from the individual harmonics at resonance, is largely determined by the acoustic losses in the "packaging material" (silicon here) and can be related to attenuation coefficient in the same media:

$Q=\frac{\pi}{\alpha \lambda}$ 

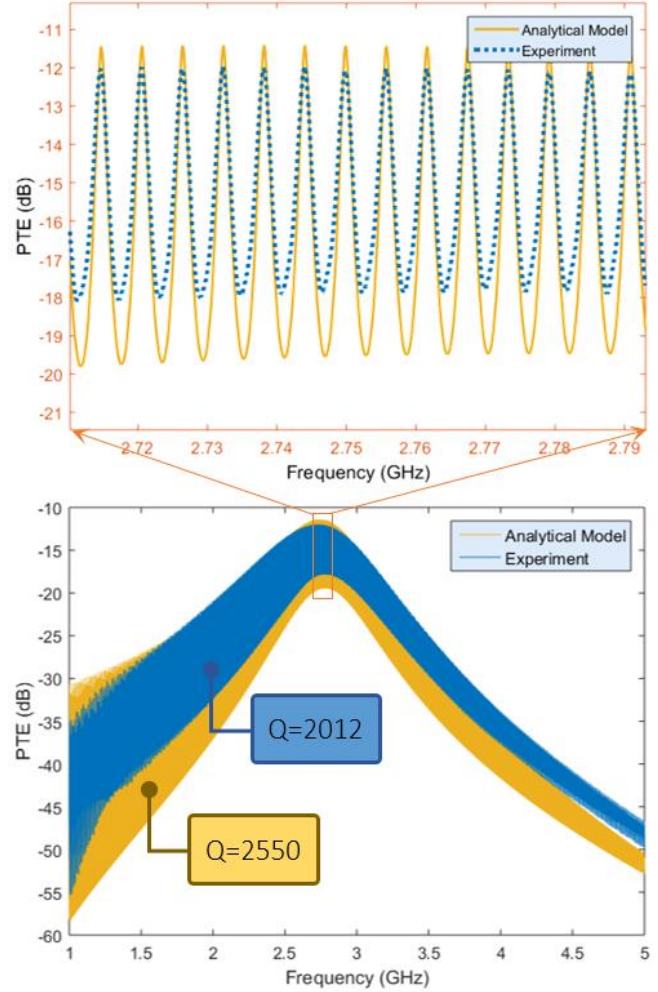

Figure 8: 2-port PTE measurement of the acoustic link.

As the results suggest, a $6 \mathrm{~dB}$ bandwidth of about $1 \mathrm{GHz}$ ensures that the acoustic link can operate efficiently over a broad range of frequencies.

\section{CONCLUSION}

This paper demonstrated the use of HBAR-like structures operating in the $\mathrm{GHz}$ range for power delivery across a package. ADS analytical simulations were conducted to predict the power gains at different frequencies and various packaging materials and thicknesses. The experimental results on the proof of concept demonstration on $700 \mu \mathrm{m}$ silicon substrate confirm accuracy of the analytical model and shows an impressive PTE of about $-12 \mathrm{~dB}$, which is due to the crystalline structure and low attenuation characteristics of silicon. Although the most frequently used nonhermetic packaging material e.g. epoxy and PDMS, have very large values of attenuation coefficients resulting in poor power gains at $\mathrm{GHz}$ range frequencies, simulation results suggest that the state-ofthe-art packaging materials such as Rexolite ${ }^{\mathbb{R}}$ can be used to effectively deliver power with acceptable PTE values. Since the attenuation coefficient of the majority of materials drops linearly with frequency, it is also expected to achieve better PTEs for thicker AlN layers (lower frequencies). Future directions include showing operation through metallic packages as well as realization of much thicker AlN films (up to $6 \mu \mathrm{m}$ ) for enhancing the acoustic channel PTE.

\section{REFERENCES}

[1] E. Mehdizadeh, et al., "Microelectromechanical Disk Resonators for Direct Detection of Liquid-Phase Analytes", Sens. Actuators Phys., 216, 1 (2014).

[2] P. J. Chen, D. C. Rodger, S. Saati, M. S. Humayun, and Y. C. Tai, "Microfabricated Implantable Parylene-Based Wireless Passive Intraocular Pressure Sensors", J. Microelectromechanical Syst., 17, 6 (2008).
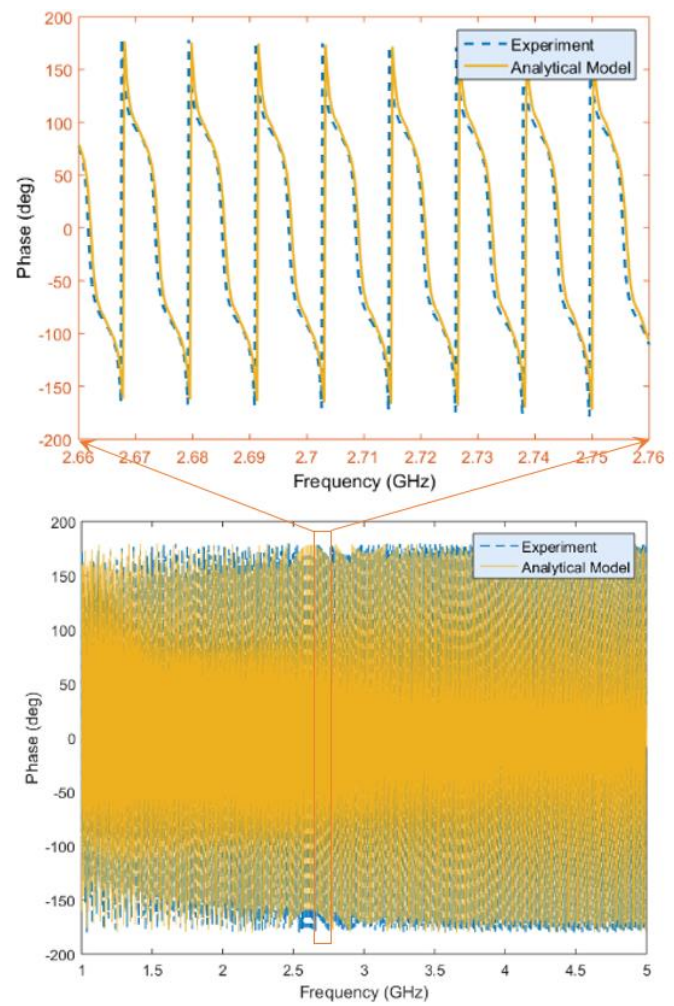

Figure 9: 2-port phase measurement of the acoustic link.

[3] E. Mehdizadeh, et al., "A two-stage aerosol impactor with embedded MEMS resonant mass balances for particulate size segregation and mass concentration monitoring," IEEE SENSORS, Baltimore (2013), pp. 1-4.

[4] D. J. Graham, J. A. Neasham, and B. S. Sharif, "Investigation of Methods for Data Communication and Power Delivery through Metals", IEEE Trans. Ind. Electron., 58, 10 (2011).

[5] M. G. L. Roes, et al., "Acoustic Energy Transfer: A Review", IEEE Trans. Ind. Electron., 60, 1 (2013).

[6] G. Piazza, et al., "Piezoelectric Aluminum Nitride Vibrating Contour-Mode MEMS Resonators", J. Microelectromechanical Syst., 15, 6, (2006).

[7] E. Defaÿ, "High Overtone Bulk Acoustic Resonator (HBAR)", in Integration of Ferroelectric and Piezoelectric Thin Films, John Wiley \& Sons, pp. 297-313, 2011.

[8] J. M. Friedt, et al., "High-Overtone Bulk Acoustic Resonator as Passive Sensor: Towards Microwave Wireless Interrogation", IEEE International Conference on Green Computing and Communications, Besançon (2012), pp. 631-636.

[9] M. Pijolat, et al., "High Quality Factor Lithium Niobate Electrostrictive HBAR Resonators", Joint Conference of the IEEE International Frequency Control and the European Frequency and Time Forum, San Francisco (2011), pp. 1-3.

[10] J. Hoople, et al., "Chip-Scale Sonic Communication using AlN Transducers", IEEE International Ultrasonics Symposium (IUS), Prague (2013), pp. 1934-1937.

[11] J. Kuo, et al., "Towards Ultrasonic through-Silicon Vias (UTSV)," IEEE International Ultrasonics Symposium, Chicago (2014), pp. 483-486.

[12] D. Royer, Elastic Waves in Solids II, Springer, New York, 2010.

[13] C. Cassella and G. Piazza, "AlN Two-Dimensional-Mode Resonators for Ultra-High Frequency Applications", IEEE Electron Device Lett., 36, 11 (2015).

\section{CONTACT}

*E. Mehdizadeh, tel: +1-720-224-4714; mehdizadeh@cmu.edu 